\title{
Efficient and Selective Synthesis of Siphonarienolone and Related Reduced Polypropionates via Zr-Catalyzed Asymmetric Carboalumination
}

\author{
Marina Magnin-Lachaux, Ze Tan, Bo Liang, and Ei-ichi Negishi*
}

\section{Supplementary Information}

General Procedures. All reactions were run under a dry Ar atmosphere. Reactions were monitored by GC analysis of reaction aliquots. GC analysis was performed on an HP6890 Gas Chromatograph using an HP-5 capillary column (30 m x $0.32 \mathrm{~mm}, 0.5 \mu \mathrm{M}$ film) packed with SE-30 on Chromosorb W. Column chromatography was carried out on 230-400 mesh silica gel. ${ }^{1} \mathrm{H}$ and ${ }^{13} \mathrm{C}$ NMR spectra were recorded on a Varian-Inova-300 spectrometer. THF was distilled from sodium/benzophenone. $\mathrm{ZnBr}_{2}$ was flame-dried under vacuum. (+)- and (-)-(NMI) $\mathrm{ZrCl}_{2},{ }^{1} \mathrm{Pd}(\mathrm{DPEphos}) \mathrm{Cl}_{2}{ }^{2}$ and 2triethylsilylpropionaldehyde- $N$-cyclohexylimine ${ }^{3}$ were prepared as reported in the literature. The starting materials were purchased from commercial sources and used as received.

(2S,4R)-2,4-Dimethylhexanal. To $1.3 \mathrm{~g}(10 \mathrm{mmol})$ of $(2 S, 4 R)$-2,4-dimethyl-1hexanol ${ }^{4}$ in $50 \mathrm{~mL}$ of $\mathrm{CH}_{2} \mathrm{Cl}_{2}$ was added consecutively $2.0 \mathrm{~g}$ of powdered $4 \AA$ molecular sieves, $100 \mathrm{mg}(0.5 \mathrm{mmol})$ of TPAP, and $2.5 \mathrm{~g}(21 \mathrm{mmol})$ of NMO. After $2 \mathrm{~h}$, the 
reaction mixtures was filtered through a short plug of silica gel with dichloromethane and concentrated. The residue was used immediately in the next step: ${ }^{1} \mathrm{H} \mathrm{NMR}\left(\mathrm{CDCl}_{3}\right) \delta$ $0.81(\mathrm{t}, J=7.0 \mathrm{~Hz}, 6 \mathrm{H}), 1.00(\mathrm{~d}, J=7.0 \mathrm{~Hz}, 3 \mathrm{H}), 1.0-1.5(\mathrm{~m}, 4 \mathrm{H}), 2.25-2.4(\mathrm{~m}, 1 \mathrm{H})$, $3.63(\mathrm{dd}, J=7.0,14.0 \mathrm{~Hz}, 1 \mathrm{H}), 9.53(\mathrm{~d}, J=2.0 \mathrm{~Hz}, 1 \mathrm{H}) ;{ }^{13} \mathrm{C} \mathrm{NMR}\left(\mathrm{CDCl}_{3}\right) \delta 11.13$, $13.22,18.62,29.69,31.67,37.14,44.14,205.36$.

$(2 E, 4 S, 6 R)-2,4,6$-Trimethyl-2-octenal $(10){ }^{5}$ Following the literature procedure, ${ }^{3}$ $s$-BuLi (5.6 mL, 1.4 M in cyclohexane, $7.8 \mathrm{mmol}$ ) was added dropwise with stirring to a solution of 2-triethylsilylpropionaldehyde- $N$-cyclohexylimine $(2.2 \mathrm{~g}, 7.8 \mathrm{mmol})$ in $16 \mathrm{~mL}$ of THF at $-78{ }^{\circ} \mathrm{C}$. After $30 \mathrm{~min}$ at $-78{ }^{\circ} \mathrm{C},(2 S, 4 R)$-2,4-dimethylhexanal $(0.77 \mathrm{~g}, 6.0$ mmol) was added. The solution was immediately warmed to $-20{ }^{\circ} \mathrm{C}$, maintained at this temperature for $2 \mathrm{~h}$, quenched with $\mathrm{H}_{2} \mathrm{O}$, and extracted 3 times with $\mathrm{Et}_{2} \mathrm{O}$. The combined organic extracts were washed with brine, dried over $\mathrm{MgSO}_{4}$, and concentrated under reduced pressure. The oil thus obtained was dissolved in $8 \mathrm{~mL}$ of dry THF and cooled to $0{ }^{\circ} \mathrm{C}$, where upon $\mathrm{CF}_{3} \mathrm{COOH}(0.83 \mathrm{~g}, 0.56 \mathrm{~mL}, 7.2 \mathrm{mmol})$ was added slowly with stirring. The reaction mixture was stirred at $0{ }^{\circ} \mathrm{C}$ for $1.5 \mathrm{~h}$, quenched with $\mathrm{H}_{2} \mathrm{O}$, maintained at $0{ }^{\circ} \mathrm{C}$ for $12 \mathrm{~h}$, and poured into saturated aqueous $\mathrm{NaHCO}_{3}$. The aqueous layer was extracted with $\mathrm{Et}_{2} \mathrm{O}$. The combined organic extracts were washed with brine, dried over $\mathrm{MgSO}_{4}$, and concentrated. The residue was purified by flash chromatography $(1 / 10$ EtOAc/hexane $)$ to provide the desired product $(0.81 \mathrm{~g}, 81 \%$ yield over 2 steps $):[\alpha]_{\mathrm{D}}=+$ $9.0^{\circ}\left(\mathrm{c} 1.5, \mathrm{CHCl}_{3}\right) ;{ }^{1} \mathrm{H} \mathrm{NMR}\left(\mathrm{CDCl}_{3}\right) \delta 0.76(\mathrm{t}, J=6.6 \mathrm{~Hz}, 6 \mathrm{H}), 0.94(\mathrm{~d}, J=6.6 \mathrm{~Hz}, 3$ H), 1.0-1.35 (m, $5 \mathrm{H}), 1.66$ (s, $3 \mathrm{H}), 2.6-2.8(\mathrm{~m}, 1 \mathrm{H}), 6.17$ (d, J = 9.9 Hz, $1 \mathrm{H}), 9.29$ (s, 1 $\mathrm{H}) ;{ }^{13} \mathrm{C} \mathrm{NMR}\left(\mathrm{CDCl}_{3}\right) \delta 9.02,10.97,19.21,19.48,29.04,30.96,31.93,43.54,137.29$, 
160.65, 195.24; IR (neat) 2962, 2929, 1691, 1642, $1456 \mathrm{~cm}^{-1}$; LRMS (EI) calcd. for $\mathrm{C}_{11} \mathrm{H}_{20} \mathrm{O} \mathrm{M}{ }^{+} 168$, found 168 .

$(3 S, 4 S, 5 E, 7 S, 9 R)$-Tetramethyl-1,5-undecadien-4-ol (11). Following the literature procedure ${ }^{6}$, trans-2-Butene $(0.28 \mathrm{~g}, 0.52 \mathrm{~mL}, 5.0 \mathrm{mmol})$ was condensed in a flame-dried graduate cylinder at $-78{ }^{\circ} \mathrm{C}$ and added to a solution of $\mathrm{KO} t$ - $\mathrm{Bu}(2.7 \mathrm{~mL}, 1 \mathrm{M}$ in THF, $2.7 \mathrm{mmol})$ at $-78{ }^{\circ} \mathrm{C} . n$-Buli $(1.1 \mathrm{~mL}, 2.5 \mathrm{M}$ in hexane, $2.7 \mathrm{mmol})$ was added slowly to the mixture while maintaining the temperature at $-78{ }^{\circ} \mathrm{C}$. The yellow mixture was warmed to $-45{ }^{\circ} \mathrm{C}$ for $30 \mathrm{~min}$, recooled to $-78{ }^{\circ} \mathrm{C}$, and a solution of dry (-)$(\mathrm{Ipc})_{2} \mathrm{BOMe}(0.84 \mathrm{~g}, 2.7 \mathrm{mmol})$ was added via cannula. After stirring at $-78{ }^{\circ} \mathrm{C}$ for 30 min, the viscous yellow mixture had totally dissolved. $\mathrm{BF}_{3} \cdot \mathrm{Et}_{2} \mathrm{O}(0.51 \mathrm{~g}, 0.45 \mathrm{~mL}, 3.6$ mmol) was added slowly, and the solution was stirred at $-78{ }^{\circ} \mathrm{C}$ until a white cloudy suspension formed. To this mixture was added $(4 S, 6 R)$-2,4,6-trimethyl-2-octenal ( $0.34 \mathrm{~g}$, $2.0 \mathrm{mmol}$ ) in $0.8 \mathrm{~mL}$ of THF via cannula. The mixture was stirred at $-78{ }^{\circ} \mathrm{C}$ over $10 \mathrm{~h}$, quenched at $-78{ }^{\circ} \mathrm{C}$ with $2 \mathrm{M} \mathrm{NaOH}$ solution $(1.3 \mathrm{~mL})$ and $30 \% \mathrm{H}_{2} \mathrm{O}_{2}$ solution $(1.7 \mathrm{~mL})$, and warmed to $23{ }^{\circ} \mathrm{C}$ with stirring under $\mathrm{Ar}$ for $20 \mathrm{~h}$. The aqueous layer was separated and extracted with EtOAc. The combined organic extracts were washed successively with $\mathrm{Na}_{2} \mathrm{SO}_{3}$ solution and brine, dried over $\mathrm{MgSO}_{4}$, and concentrated in vacuo. The crude mixture was purified by flash chromatography in hexane followed by $5 \%$ EtOAc/hexane to afford $383 \mathrm{mg}$ of the desired product in $85 \%$ yield $($ anti/syn $=10 / 1=91 / 9):[\alpha]_{\mathrm{D}}=+2^{\circ}$ (c 1.6, $\left.\mathrm{CHCl}_{3}\right) ;{ }^{1} \mathrm{H}$ NMR $\left(\mathrm{CDCl}_{3}\right) \delta$ 0.75-0.9 (m, $\left.9 \mathrm{H}\right), 0.95-1.1(\mathrm{~m}, 3 \mathrm{H}), 1.1-1.4(\mathrm{~m}, 5 \mathrm{H})$, $1.59(\mathrm{~s}, 3 \mathrm{H}), 1.70(\mathrm{~s}, 1 \mathrm{H}), 2.2-2.35(\mathrm{~m}, 1 \mathrm{H}), 2.4-2.55(\mathrm{~m}, 1 \mathrm{H}), 3.58(\mathrm{~d}, J=8.9 \mathrm{~Hz}, 1$ $\mathrm{H})$, 5.05-5.2 (m, $3 \mathrm{H}), 5.65-5.8(\mathrm{~m}, 1 \mathrm{H}) ;{ }^{13} \mathrm{C} \mathrm{NMR}\left(\mathrm{CDCl}_{3}\right) \delta 10.79,11.19,16.81,19.56$, 
$20.82,28.96,29.60,31.99,42.20,44.79,81.68,116.31,132.65,136.35,141.51$; IR (neat)

3446, 2960, 2926, 1717, 1668, 1638, 1456, $1377 \mathrm{~cm}^{-1}$; LRMS (EI) calcd. for $\mathrm{C}_{15} \mathrm{H}_{28} \mathrm{O} \mathrm{M}^{+}$ 224, found 224 .

\section{Siphonarienal $((2 E, 4 S, 6 S, 8 S)-2,4,6,8$-Tetramethyl-2-undecenal $) \quad(3){ }^{7}$ To a} stirred solution of oxalyl chloride $(0.30 \mathrm{~g}, 0.21 \mathrm{~mL}, 2.4 \mathrm{mmol})$ in $9 \mathrm{~mL}$ of $\mathrm{CH}_{2} \mathrm{Cl}_{2}$ at -78 ${ }^{\circ} \mathrm{C}$ was added dropwise via cannula DMSO $(0.38 \mathrm{~g}, 0.34 \mathrm{~mL}, 4.8 \mathrm{mmol})$ in $3 \mathrm{~mL}$ of $\mathrm{CH}_{2} \mathrm{Cl}_{2}$. After $30 \mathrm{~min}$ stirring at $-78{ }^{\circ} \mathrm{C},(2 \mathrm{~S}, 4 \mathrm{~S}, 6 \mathrm{~S})-2,4,6$-trimethyl-1-nonanol ${ }^{4}(0.38 \mathrm{~g}$, $2.0 \mathrm{mmol}$ ) in $2 \mathrm{~mL}$ of $\mathrm{CH}_{2} \mathrm{Cl}_{2}$ was added slowly via cannula maintaining. The resulting white heterogeneous mixture was stirred at $-78{ }^{\circ} \mathrm{C}$ for $2 \mathrm{~h}$, and $\mathrm{Et}_{3} \mathrm{~N}(0.61 \mathrm{~g}, 0.84 \mathrm{~mL}, 6.0$ mmol) was added. After $5 \mathrm{~min}$ at $-78{ }^{\circ} \mathrm{C}$, the mixture was warmed to $23{ }^{\circ} \mathrm{C}$ for $1.5 \mathrm{~h}$ and $4 \mathrm{~mL}$ of $\mathrm{H}_{2} \mathrm{O}$ was added. The aqueous layer was extracted with $\mathrm{CH}_{2} \mathrm{Cl}_{2}$. The combined organic extracts were washed with brine, dried over $\mathrm{MgSO}_{4}$, and concentrated under reduced pressure. The crude residue was used immediately for the next step.

$s$ - BuLi (1.9 mL, 1.4 M in pentane, $2.6 \mathrm{mmol})$ was added dropwise with stirring to a solution of 2-triethylsilylpropionaldehyde- $N$-cyclohexylimine $(0.66 \mathrm{~g}, 2.6 \mathrm{mmol})$ in THF $(5.2 \mathrm{~mL})$ at $-78{ }^{\circ} \mathrm{C}$. After $30 \mathrm{~min}$ at $-78{ }^{\circ} \mathrm{C}$, the above-prepared crude $(2 S, 4 S, 6 S)$ 2,4,6-trimethyl-1-nonanal ( $0.37 \mathrm{~g}, 2.0 \mathrm{mmol})$ in $2 \mathrm{~mL}$ of THF was added to the reaction mixture. The solution was immediately warmed to $-20{ }^{\circ} \mathrm{C}$, and maintained at this temperature for $2 \mathrm{~h}$. The reaction mixture was quenched with $\mathrm{H}_{2} \mathrm{O}$ and extracted with $\mathrm{Et}_{2} \mathrm{O}$. The combined organic extracts were washed with brine, dried over $\mathrm{MgSO}_{4}$, and concentrated under reduced pressure. The oil thus obtained was dissolved in dry THF (2.8 mL) and cooled to $0{ }^{\circ} \mathrm{C}$, where upon $\mathrm{CF}_{3} \mathrm{COOH}(0.27 \mathrm{~g}, 0.18 \mathrm{~mL}, 2.4 \mathrm{mmol})$ was 
added slowly. After $1.5 \mathrm{~h}$ at $0{ }^{\circ} \mathrm{C}, \mathrm{H}_{2} \mathrm{O}(2 \mathrm{~mL})$ was added, and the resultant mixture was maintained at $0{ }^{\circ} \mathrm{C}$ for another $2 \mathrm{~h}$. The brown mixture was poured into saturated $\mathrm{NaHCO}_{3}$, and the aqueous layer was extracted with $\mathrm{Et}_{2} \mathrm{O}$. The combined organic extracts were washed with brine, dried over $\mathrm{MgSO}_{4}$, and concentrated. The resulting residue was chromatographed (hexane/EtOAc $10 / 1)$ to provide the desired product $(0.38 \mathrm{~g}, 86 \%$ yield (2 steps)): ${ }^{1} \mathrm{H}$ NMR $\left(\mathrm{CDCl}_{3}\right) \delta$ 0.65-0.85 (m, $\left.9 \mathrm{H}\right), 0.85-0.95(\mathrm{~m}, 1 \mathrm{H}), 0.98(\mathrm{~d}, J=6.6$ Hz, $3 \mathrm{H}), 1.0-1.5$ (m, 9 H), $1.70(\mathrm{~s}, 3 \mathrm{H}), 2.7-2.85(\mathrm{~m}, 1 \mathrm{H}), 6.14(\mathrm{~d}, J=10.0 \mathrm{~Hz}, 1 \mathrm{H})$, $9.32(\mathrm{~s}, 1 \mathrm{H}) ;{ }^{13} \mathrm{C} \mathrm{NMR}\left(\mathrm{CDCl}_{3}\right) \delta$ 9.23, 14.25, 19.87, 19.91, 20.21, 20.41, 28.11, 29.50, 31.11, 39.13, 44.10, 45.47, 137.86, 160.47, 195.28; IR (neat) 2958, 2705, 1691, 1643, $1457 \mathrm{~cm}^{-1}$; HRMS calcd. for $\mathrm{C}_{15} \mathrm{H}_{28} \mathrm{O}[\mathrm{M}+\mathrm{H}]^{+} 225.2219$, found 225.2215 .

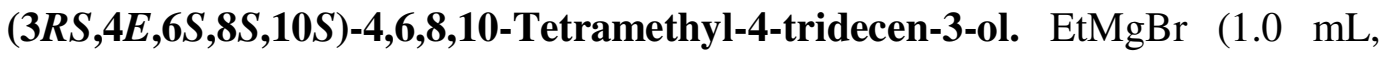
3.0 $\mathrm{M}$ in $\mathrm{Et}_{2} \mathrm{O}, 3.0 \mathrm{mmol}$ ) was added dropwise with stirring to a solution of siphonarienal $(0.34 \mathrm{~g}, 1.5 \mathrm{mmol})$ in $\mathrm{THF}(6 \mathrm{~mL})$ at $-78{ }^{\circ} \mathrm{C}$. The reaction mixture was maintained at -78 ${ }^{\circ} \mathrm{C}$ for $30 \mathrm{~min}$, quenched with $\mathrm{H}_{2} \mathrm{O}$ and warmed to $23{ }^{\circ} \mathrm{C}$. The aqueous phase was separated and extracted with $\mathrm{Et}_{2} \mathrm{O}$. The combined organic extracts were washed with saturated $\mathrm{NH}_{4} \mathrm{Cl}$, brine, dried over $\mathrm{MgSO}_{4}$, and concentrated. The residue was purified by column chromatography ( $1 \%$ to $10 \%$ EtOAc/hexane) to afford the title compound (0.34 g, 87\% yield): ${ }^{1} \mathrm{H}$ NMR $\left(\mathrm{CDCl}_{3}\right) \delta$ 0.7-0.85 (m, $\left.10 \mathrm{H}\right), 0.86(\mathrm{t}, J=6.4 \mathrm{~Hz}, 3 \mathrm{H}), 0.9-1.05$ (m, $3 \mathrm{H}), 1.05-1.35(\mathrm{~m}, 6 \mathrm{H}), 1.35-1.5(\mathrm{~m}, 3 \mathrm{H}), 1.5-1.6(\mathrm{~m}, 3 \mathrm{H}), 1.58$ (s, $3 \mathrm{H}), 2.4-2.55$ $(\mathrm{m}, 1 \mathrm{H}), 3.69(\mathrm{t}, J=6.6 \mathrm{~Hz}, 1 \mathrm{H}), 5.07(\mathrm{~d}, J=9.5 \mathrm{~Hz}, 1 \mathrm{H}) ;{ }^{13} \mathrm{C} \mathrm{NMR}\left(\mathrm{CDCl}_{3}\right) \delta 9.92$, $11.60,14.38,19.98,20.08,20.47,21.78,27.63,27.91,29.51,29.61,39.34,44.97,45.71$, 
79.11, 133.21, 135.21; IR (neat) 3359, 1457, $1377 \mathrm{~cm}^{-1}$; LRMS (EI) calcd. for $\mathrm{C}_{17} \mathrm{H}_{34} \mathrm{O}$ $\mathrm{M}^{+} 254$, found 254 .

Siphonarienone $((4 E, 6 S, 8 S, 10 S)-4,6,8,10-T e t r a m e t h y l-4-t r i d e c e n-3-o n e) ~(2) .{ }^{8}$ To a solution of Dess-Martin periodinane $(0 . .68 \mathrm{~g}, 1.6 \mathrm{mmol})$ in $3 \mathrm{~mL}$ of $\mathrm{CH}_{2} \mathrm{Cl}_{2}$ and 1 $\mathrm{mL}$ pyridine was added $(3 R S, 4 E, 6 S, 8 S, 10 S)$-4,6,8,10-tetramethyl-4-tridecen-3-ol (0.26 g, $1.0 \mathrm{mmol}$ ) diluted in $1.5 \mathrm{~mL}$ of $\mathrm{CH}_{2} \mathrm{Cl}_{2}$ at $23{ }^{\circ} \mathrm{C}$. After $1.5 \mathrm{~h}$, the reaction mixture was diluted with $\mathrm{CH}_{2} \mathrm{Cl}_{2}$, washed successively with $\mathrm{H}_{2} \mathrm{O}, 0.5 \mathrm{~N} \mathrm{HCl}$, saturated $\mathrm{NaHCO}_{3}$, and brine. The solvent was dried over $\mathrm{MgSO}_{4}$ and concentrated. The residue was purified by column chromatography (EtOAc/hexane 3\%) to afford $234 \mathrm{mg}$ of the desired product in $91 \%$ yield: $[\alpha]_{\mathrm{D}}=+26.4^{\mathrm{o}}\left(\mathrm{c} 1.4, \mathrm{CHCl}_{3}\right) ;{ }^{1} \mathrm{H} \mathrm{NMR}\left(\mathrm{CDCl}_{3}\right) \delta 0.76(\mathrm{~d}, J=6.9 \mathrm{~Hz}, 3 \mathrm{H})$, $0.79(\mathrm{~d}, J=6.9 \mathrm{~Hz}, 3 \mathrm{H}), 0.83(\mathrm{t}, J=6.9 \mathrm{~Hz}, 3 \mathrm{H}), 0.96(\mathrm{~d}, J=6.6 \mathrm{~Hz}, 3 \mathrm{H}), 1.05(\mathrm{t}, J=$ $7.5 \mathrm{~Hz}, 3 \mathrm{H}) ; 1.1-1.5(\mathrm{~m}, 10 \mathrm{H}), 1.76(\mathrm{~s}, 3 \mathrm{H}), 2.55-2.75$ (m, $1 \mathrm{H}), 2.63$ (q, $J=7.5 \mathrm{~Hz}, 2$ $\mathrm{H}), 6.29(\mathrm{~d}, J=9.8 \mathrm{~Hz}, 1 \mathrm{H}){ }^{13} \mathrm{C} \mathrm{NMR}\left(\mathrm{CDCl}_{3}\right) \delta 8.91,11.45,14.32,19.91(2 \mathrm{C}), 20.38$, $20.65,28.20,29.57,30.29,31.18,39.25,44.40,45.50,135.30,148.15,202.71$. HRMS calcd. for $\mathrm{C}_{17} \mathrm{H}_{32} \mathrm{O}[\mathrm{M}+\mathrm{H}]^{+}$253.2532, found 253.2526.

\section{$(2 S, 4 R, 5 S, 6 E, 8 S, 10 S, 12 S)$-2-Benzoyloxy-5-hydroxy-4,6,8,10,12-pentamethyl-}

pentadec-6-en-3-one (8). To a solution of $c$ - $\mathrm{Hex}_{2} \mathrm{BCl}(0.37 \mathrm{~g}, 0.39 \mathrm{~mL}, 1.8 \mathrm{mmol})$ in $\mathrm{Et}_{2} \mathrm{O}(5 \mathrm{~mL})$ was added $\mathrm{Me}_{2} \mathrm{NEt}(0.16 \mathrm{~g}, 0.23 \mathrm{~mL}, 2.1 \mathrm{mmol})$. A solution of the $(2 S)$ benzoyloxy-3-pentanone $(0.24 \mathrm{~g}, 1.2 \mathrm{mmol})$ in $\mathrm{Et}_{2} \mathrm{O}(2 \mathrm{~mL})$ was added $-78{ }^{\circ} \mathrm{C}$. The resultant suspension was stirred $10 \mathrm{~min}$ at $-78{ }^{\circ} \mathrm{C}, 2 \mathrm{~h}$ at $0{ }^{\circ} \mathrm{C}$, and recooled to $-78{ }^{\circ} \mathrm{C}$. A solution of freshly prepared siphonarienal $(0.22 \mathrm{~g}, 1.2 \mathrm{mmol})$ in $\mathrm{Et}_{2} \mathrm{O}(2 \mathrm{~mL})$ was added. After $1 \mathrm{~h}$ at $-78{ }^{\circ} \mathrm{C}$, the solution was placed in the freezer overnight $\left(-20^{\circ} \mathrm{C}\right)$. The reaction 
mixture was warmed to $0{ }^{\circ} \mathrm{C}$ and quenched with a mixture of $\mathrm{MeOH}(2 \mathrm{~mL})$ and $\mathrm{pH} 7$ buffer $(4 \mathrm{~mL})$ followed by $30 \%$ aq $\mathrm{H}_{2} \mathrm{O}_{2}(2 \mathrm{~mL})$. After $1 \mathrm{~h}$ at $0{ }^{\circ} \mathrm{C}$, the layers were separated, and the aqueous layer was extracted with $\mathrm{Et}_{2} \mathrm{O}$. The combined organic extracts were dried over $\mathrm{MgSO} 4$, filtered, and concentrated. The residue was purified by column chromatography (5\% to $8 \%$ EtOAc/hexane) to afford the title compound $(0.35 \mathrm{~g}, 82 \%$ yield): ${ }^{1} \mathrm{H}$ NMR $\left(\mathrm{CDCl}_{3}\right) \delta$ 0.7-1.0 (m, $\left.16 \mathrm{H}\right), 1.0-1.5(\mathrm{~m}, 9 \mathrm{H}), 1.55(\mathrm{~d}, J=7.1 \mathrm{~Hz}, 3 \mathrm{H})$, 1.59 (s, $3 \mathrm{H}), 1.85-2.05$ (bs, $1 \mathrm{H}), 2.4-2.6(\mathrm{~m}, 1 \mathrm{H}), 2.95-3.1(\mathrm{~m}, 1 \mathrm{H}), 4.16(\mathrm{~d}, J=9.5 \mathrm{~Hz}$, $1 \mathrm{H}), 5.10(\mathrm{~d}, J=9.5 \mathrm{~Hz}, 1 \mathrm{H}), 5.43(\mathrm{q}, J=7 \mathrm{~Hz}, 1 \mathrm{H}), 7.42(\mathrm{t}, J=7.3 \mathrm{~Hz}, 2 \mathrm{H}), 7.55(\mathrm{t}, J$ $=7.3 \mathrm{~Hz}, 1 \mathrm{H}), 8.05(\mathrm{~d}, J=8.0 \mathrm{~Hz}, 2 \mathrm{H}) ;{ }^{13} \mathrm{C} \mathrm{NMR}\left(\mathrm{CDCl}_{3}\right) \delta 10.35,14.36,14.42,15.51$, $19.95,20.02,20.04,21.63,28.09,29.54,29.65,39.21,44.80,45.28,45.81,75.14,80.58$, 128.36 (2C), 129.59, 129.75 (2C), 132.56, 133.18, 136.92, 165.83, 210.95.

\section{$(2 S, 4 R, 5 S, 6 E, 8 S, 10 S, 12 S)$-2-Benzoyloxy-5-(tert-butyldimethylsilyloxy)-}

4,6,8,10,12-pentamethyl-pentadec-6-en-3-one. To a cooled $\left(-78{ }^{\circ} \mathrm{C}\right)$ solution of the aldol product $(2 S, 4 R, 5 S, 6 E, 8 S, 10 S, 12 S)$-2-Benzoyloxy-5-hydroxy-4,6,8,10,12pentamethyl-pentadec-6-en-3-one ( $0.22 \mathrm{~g}, 0.50 \mathrm{mmol})$ in $\mathrm{CH}_{2} \mathrm{Cl}_{2}(3 \mathrm{~mL})$ was added 2, 6lutidine $(0.21 \mathrm{~g}, 2.0 \mathrm{mmol})$ followed by TBSOTf $(0.40 \mathrm{~g}, 1.5 \mathrm{mmol})$. After $1 \mathrm{~h}$ and 15 min, TLC analysis showed complete consumption of the starting material. The reaction mixture was quenched with saturated $\mathrm{NaHCO}_{3}$ solution $(10 \mathrm{~mL})$, warmed to $23{ }^{\circ} \mathrm{C}$, and partitioned between $\mathrm{H}_{2} \mathrm{O}(10 \mathrm{~mL})$ and $\mathrm{CH}_{2} \mathrm{Cl}_{2}(3 \times 25 \mathrm{~mL})$. The combined organic extracts were dried over $\mathrm{MgSO}_{4}$, concentrated under vacuum, and purified by column chromatography to affored the desired product $(0.27 \mathrm{~g}, 99 \%$ yield $) .{ }^{1} \mathrm{H} \mathrm{NMR}\left(\mathrm{CDCl}_{3}\right) \delta$ 0.05 (s, $6 \mathrm{H}), 0.7-1.0(\mathrm{~m}, 26 \mathrm{H}), 1.0-1.5(\mathrm{~m}, 8 \mathrm{H}), 1.51(\mathrm{~d}, J=7.1 \mathrm{~Hz}, 3 \mathrm{H}), 1.55(\mathrm{~s}, 3 \mathrm{H})$, 
2.4-2.6 (m, $1 \mathrm{H}), 2.95-3.1(\mathrm{~m}, 1 \mathrm{H}), 4.19(\mathrm{~d}, J=9.7 \mathrm{~Hz}, 1 \mathrm{H}), 5.02(\mathrm{~d}, J=9.7 \mathrm{~Hz}, 1 \mathrm{H})$, $5.42(\mathrm{q}, J=6.9 \mathrm{~Hz}, 1 \mathrm{H}), 7.4-7.6(\mathrm{~m}, 3 \mathrm{H}), 8.06(\mathrm{~d}, J=7.3 \mathrm{~Hz}, 2 \mathrm{H}) ;{ }^{13} \mathrm{C} \mathrm{NMR}\left(\mathrm{CDCl}_{3}\right)$ $\delta-5.09,-4.61,10.26,14.39,14.53,15.18,18.04,19.93,19.99,20.07,21.21,25.81(3 \mathrm{C})$, $28.12,29.58,29.66,39.29,44.83,45.92,45.97,75.34,81.77,128.36$ (2C), 129.77, 129.80 (2C), 132.76, 133.14, 136.39, 165.70, 209.50. IR (neat) 2957, 1724, 1456, 1267 $\mathrm{cm}^{-1}$.

$(4 R, 5 S, 6 E, 8 S, 10 S, 12 S)$-5-(tert-Butyldimethylsilyloxy)-4,6,8,10,12-

pentamethyl-pentadec-6-en-3-one (9). To a cooled $\left(0{ }^{\circ} \mathrm{C}\right)$ solution of the protected adol product $(0.22 \mathrm{~g}, 0.4 \mathrm{mmol})$ in THF $(4.8 \mathrm{~mL})$ and $\mathrm{MeOH}(2.4 \mathrm{~mL})$ was added $\mathrm{SmI}_{2}(13$ $\mathrm{mL}, 0.1 \mathrm{M}$ solution in THF) until a green color persisted in the reaction mixture. After 30 min, saturated $\mathrm{K}_{2} \mathrm{CO}_{3}$ solution $(24 \mathrm{~mL})$ was added and the mixture was extracted with ether. The combined organic layers were dried over $\mathrm{MgSO}_{4}$, concentrated under reduced pressure, and purified by column chromatography (5\% EtOAc/hexane) to give $0.16 \mathrm{~g}$ of the title compound in 94\% yield: ${ }^{1} \mathrm{H}$ NMR $\left(\mathrm{CDCl}_{3}\right) \delta-0.08(\mathrm{~s}, 6 \mathrm{H}), 0.7-1.0(\mathrm{~m}, 29 \mathrm{H})$, 1.0-1.55 (m, $8 \mathrm{H}), 1.54$ (s, $3 \mathrm{H}), 2.4-2.6(\mathrm{~m}, 3 \mathrm{H}), 2.7-2.85(\mathrm{~m}, 1 \mathrm{H}), 4.02(\mathrm{~d}, J=9.8 \mathrm{~Hz}$,

$1 \mathrm{H}), 5.00(\mathrm{~d}, J=9.7 \mathrm{~Hz}, 1 \mathrm{H}) ;{ }^{13} \mathrm{C}$ NMR $\left(\mathrm{CDCl}_{3}\right) \delta-5.46,-4.54,7.24,10.20,13.89$, $14.39,17.96,19.78,20.00,20.13,21.25,25.70$ (3C), 28.11, 29.60, 29.64, 38.02, 39.20, $44.97,46.03,48.72,82.76,133.08,136.00,215.01$.

Siphonarienolone.

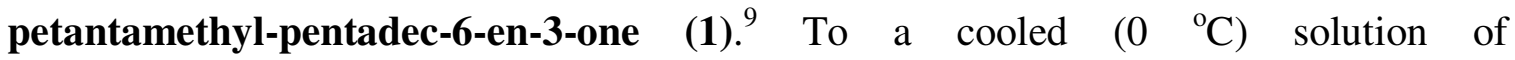
$(4 R, 5 S, 6 E, 8 S, 10 S, 12 S)$-5-(tert-butyldimethylsilyloxy)-4,6,8,10,12-pentamethyl-pentadec- 
6-en-3-one (24 mg, $0.056 \mathrm{mmol})$ in THF $(4.8 \mathrm{~mL})$ in a TEFLON tube was added HF.Pyridine $(0.96 \mathrm{~mL})$. The reaction mixture was stirred $1 \mathrm{~h}$ at $0{ }^{\circ} \mathrm{C}$ and $6 \mathrm{~h}$ at $23{ }^{\circ} \mathrm{C}$. $\mathrm{H}_{2} \mathrm{O}(5 \mathrm{~mL})$ was added and the mixture was extracted with ether. The combined organic layers were washed with saturated $\mathrm{NaHCO}_{3}$, dried over $\mathrm{MgSO}_{4}$, and concentrated. Column chromatography on silica gel (8-20\% EtOAc/hexane) afforded $15 \mathrm{mg}$ of the desired compound in $87 \%$ yield: $\geq 50 / 1 \mathrm{dr} ;[\alpha]_{\mathrm{D}}=+11^{\circ}\left(\mathrm{c} 0.3, \mathrm{CHCl}_{3}\right)$, lit. $[\alpha]_{\mathrm{D}}=+19.6^{\circ}$

$\left(\mathrm{c} 0.9, \mathrm{CHCl}_{3}\right) ;{ }^{1} \mathrm{H} \mathrm{NMR}\left(\mathrm{CDCl}_{3}\right) \delta 0.6-0.9(\mathrm{~m}, 20 \mathrm{H}), 1.03(\mathrm{t}, J=7.4 \mathrm{~Hz}, 3 \mathrm{H}), 1.1-1.5$ (m, 5 H), 1.60 (s, 3 H), 1.75-2.1 (bs, $1 \mathrm{H}), 2.4-2.6$ (m, $3 \mathrm{H}), 2.75-2.8$ (m, $1 \mathrm{H}), 4.08$ (d, J $=9.2 \mathrm{~Hz}, 1 \mathrm{H}), 5.10(\mathrm{~d}, J=9.6 \mathrm{~Hz}, 1 \mathrm{H}) ;{ }^{13} \mathrm{C} \mathrm{NMR}\left(\mathrm{CDCl}_{3}\right) \delta$ 7.48, 10.58, 14.19, 14.40, $19.98,20.10$ (2 C), 21.67, 28.12, 29.59, 29.67, 36.32, 39.20, 44.93, 45.86, 48.42, 80.76, 132.73, 136.60, 215.84. HRMS calcd. for $\mathrm{C}_{20} \mathrm{H}_{38} \mathrm{O}_{2}[\mathrm{M}+\mathrm{H}]^{+}$311.2951, found 311.2948.

\section{Reference:}

1. Erker, G.; Aulbach, M.; Knickmeier, M.; Wingbermuhle, D.; Kruger, C.; Nolte, M.; Werner, S. J. Am. Chem. Soc. 1993, 115, 4590.

2. Kranenburg, M.; van der Burgt, Y. E. M.; Kamer, P. C. J.; van Leeuwen, P. W. N. M.; Goubitz, K.; Fraanje, J. Organometallics 1995, 14, 3081.

3. Corey, E. J.; Enders, D.; Bock, M. G. Tetrahedron Lett. 1976, 1, 7.

4. Negishi, E.; Tan, Z.; Liang, B.; Novak, T. Proc. Natl. Acad. Sci. in press.

5. Williams, D. R.; Turske, R. A. Org. Lett. 2000, 2, 3217.

6. Brown, H. C.; Bhat, K. S. J. Am. Chem. Soc. 1986, 108, 5919.

7. Calter, M. A.; Liao, W.; Struss, J. A. J. Org. Chem. 2001, 66, 7500.

8. Norte, M.; Cataldo, F.; Gonzalez, A. G.; Rodriguez, M. L.; Ruiz-Perez, C. Tetrahedron 1990, 46, 1669.

9. Calter, M. A.; Liao, W. J. Am. Chem. Soc. 2002, 124, 13127. 